\title{
Collectif, The Art of Medieval Hungary
}

\section{Cécile Voyer}

\section{(2) OpenEdition}

\section{Journals}

Édition électronique

URL : https://journals.openedition.org/ccm/8646

DOI : $10.4000 / \mathrm{ccm} .8646$

ISSN : 2119-1026

\section{Éditeur}

Centre d'études supérieures de civilisation médiévale/Université de Poitiers

\section{Édition imprimée}

Date de publication : 1 décembre 2021

Pagination : 367-369

ISBN : $978-2-490783-11-3$

ISSN : 0007-9731

Référence électronique

Cécile Voyer, "Collectif, The Art of Medieval Hungary », Cahiers de civilisation médiévale [En ligne], 256 | 2021, mis en ligne le 01 décembre 2021, consulté le 25 mai 2022. URL : http:// journals.openedition.org/ccm/8646; DOI : https://doi.org/10.4000/ccm.8646

La revue Cahiers de civilisation médiévale est mise à disposition selon les termes de la Licence Creative Commons Attribution - Pas d'Utilisation Commerciale - Pas de Modification 4.0 International. 


\section{COMPTES RENDUS}

The Art of Medieval Hungary, Xavier Barral i Altet, Pál Lövei, Vinni Lucherini et Imre Takács (éd.), Rome, Viella (Bibliotheca academiae hungariae - Roma, Studia, 7), 2018.

Rares sont les entreprises éditoriales et scientifiques de cette nature, qui visent à la fois la synthèse et la réflexion historiographique. Soutenu par l'Académie hongroise de Rome et l'Académie des sciences de Bucarest, cet ouvrage sur l'art de la Hongrie médiévale en est une véritable illustration.

Si l'ambition de ce volume n'est pas de poursuivre le projet éditorial des grandes collections de la première moitié du $\mathrm{xx}^{\mathrm{e}} \mathrm{s}$., marqué par des préoccupations qui ne sont plus les nôtres, elle propose de renouer avec la tradition de la synthèse en histoire de l'art comme objet intellectuel et proposition scientifique.

Ce travail collectif dirigé par Xavier Barral i Altet, Pàl Lővei, Vinni Lucherini, Imre Takàcs n'est pas non plus un manuel, c'est aussi un outil réflexif. L'ouvrage tend en effet à poser les bases de la recherche sur les arts en Hongrie médiévale pour les prochaines décennies. Une telle démarche nécessitait donc une importante réflexion historiographique; réflexion historiographique qui a présidé au choix même du titre du livre - «L'art de la Hongrie médiévale » plutôt que «L'art en Hongrie médiévale », les auteurs s'étant d'ailleurs bien gardé de l'emploi de dangereuses épithètes " L'art médiéval hongrois 》- car l'art de cette entité politique y est appréhendé dans une perspective résolument européenne. Grâce aux résultats de la recherche la plus récente, l'ouvrage éclaire la création et la production artistiques au cœur de l'Europe médiane, entre la fin du $\mathrm{X}^{\mathrm{e}} \mathrm{s}$. (la fondation de l'état chrétien de Hongrie par Étienne I ${ }^{\mathrm{er}}$ ) et le $\mathrm{Xv}^{\mathrm{e}} \mathrm{s}$. Ses contributeurs y observent les relations artistiques entre la Hongrie médiévale, en expansion, et les autres aires culturelles européennes. En le pensant à cette échelle, les auteurs offrent ainsi un vaste panorama de l'ensemble de la production artistique de la Hongrie médiévale, loin du système épistémologique fermé qui a contribué à la fabrique de l'art national. L'utile mise au point de X. Barral dans l'introduction permet de donner le ton. La géographie de l'art qui a façonné l'histoire de l'art des différents pays européens, entre la fin du $\mathrm{XIX}^{\mathrm{e}} \mathrm{s}$. et la première moitié du $\mathrm{XX}^{\mathrm{e}} \mathrm{s}$., a d'ailleurs continué, malgré son renouvellement, à alimenter les débats, et ce dès les années 1970 (Enrico Castelnuovo et Carlo Ginzburg, « Domination symbolique et géographie artistique dans l'histoire de l'art italien ", Actes de la recherche en sciences sociales, « Sociologie de l'œil », 40, 1981, p. 51-72, DOI : 10.3406/arss.1981.2133). Pour ces raisons, X. Barral invite à réfléchir non seulement à la terminologie utilisée par les historiens de l'art (la notion de centre et de périphérie par exemple), mais aussi aux critères choisis pour définir la production artistique d'un territoire donné au cours d'un temps plus ou moins long. Les critères spatiaux (aire culturelle ou géographique) et temporels (historique, politique, dynastique...) participent toujours d'une construction qui doit être soumise à un examen critique. L'utilisation du terme hongrois est également l'objet d'un questionnement. Anna Boreczky lui préfère l'adjectif « indigène » plus approprié pour exprimer la diversité ethnique des populations qui vivent dans le royaume magyar, l'épithète d'hongrois servant plutôt à qualifier l'entité politique aux frontières mouvantes au cours des $\mathrm{XII}^{\mathrm{e}}$-XIII ${ }^{\mathrm{e}} \mathrm{s}$. (p. 284). Le royaume de Hongrie au Moyen Âge s'avère être un terrain d'exploration privilégié pour mener à bien cette réflexion méthodologique et épistémologique. Il conservait en effet des spécificités, tout en étant ouvert aux autres aires culturelles et/ou politiques (Pologne, Bohême et Croatie mais aussi France capétienne, Italie, Empire germanique et monde byzantin). Au tournant des $\mathrm{XI}^{\mathrm{e}}-\mathrm{XII}^{\mathrm{e}}$ s., la création artistique y était de surcroît favorisée par de bonnes conditions politiques - un pouvoir puissant -, économiques et sociales. Afin de mesurer les continuités et les variations, la création et la production artistiques sont envisagées sur le temps long $\left(\mathrm{X}^{\mathrm{e}}-\mathrm{XV}^{\mathrm{e}} \mathrm{s}\right.$. $)$, cher à 
Jacques Le Goff. Pas plus que le territoire, l'histoire n'est ici découpée en tranches (Jacques Le GofF, Faut-il vraiment découper l'histoire en tranches?, Paris, Seuil [La librairie du XxI ${ }^{\mathrm{e}}$ siècle], 2014).

Le projet d'ensemble se décline en une série de thématiques nourries par l'actualité de la recherche et les questionnements historiographiques. Ces sections qui servent à définir l'art au sein de cette aire politique sont accompagnées d'une importante bibliographie, véritable outil pour les recherches futures. Le choix d'une contextualisation systématique de la production artistique explique les différents chapitres de l'ouvrage (phénomènes historico-politiques, cadre liturgique et rituel, constructions sociales et culturelles, construction historiographique).

Le premier chapitre est une nécessaire présentation des sources et des travaux sur l'art médiéval du royaume de Hongrie dans une perspective historiographique ("Sources and Studies for Hungarian Medieval Art ", E. Marosi et K. SzovàK). La deuxième section du livre, "City and Territory » (K. Szende, P. Lővei, Zs. Jékely et I. Feld), en utilisant le concept de continuité et de discontinuité est une observation fine du développement du fait urbain (villes et réseaux urbains dans le bassin des Carpates) avec une analyse de la construction urbaine et de ses ouvrages défensifs tout en en soulignant les spécificités architecturales. Selon une démarche similaire, l'observation de la mise en place du système ecclésial et de son quadrillage du territoire au $\mathrm{XI}^{\mathrm{e}} \mathrm{s}$. offre une étude sur le rôle social des églises paroissiales avec une réflexion sur l'édification des lieux de culte, la réalisation de leur décor et leurs ornements liturgiques. Il aurait sans doute été intéressant de convoquer le concept d'inecclesiamento, défendu avec brio par Michel Lauwers pour qualifier ce phénomène lent et graduel. Cette notion vise à souligner en effet le rôle primordial de l'Ecclesia par la construction des églises et la sacralisation d'une zone jouxtant l'édifice et délimitée par le circuitus. La prise en compte de différents paramètres sociaux, politiques et conjoncturels permet d'expliquer, à la fin du Moyen Âge, la construction des églises fortifiées avec, parfois, des lieux de stockage pour les récoltes du village. Dans l'organisation des territoires, le rôle défensif, stratégique et politique des châteaux, forteresses et manoirs, n'a pas été oublié.

Le troisième chapitre offre un vaste panorama sur l'architecture religieuse, les images et les objets dans l'expérience liturgique («Architecture and Art in the Context of Liturgy »). Si pour traiter ce thème, les auteurs ont opté pour la séparation classique entre art roman et art gothique, la contextualisation précise des exemples cités (les patronages, le rôle des communautés religieuses, le goût des élites) permet de comprendre leurs intentions (B. Zs. Szakàcs, K. Havasi, I. Takàcs, P. Lővei, I. Takàcs et G. Endrödi). Sur le long temps, il est possible d'observer le tropisme vers Byzance, puis des orientations culturelles vers la France capétienne avec la dynastie arpadienne ( $\mathrm{X}^{\mathrm{e}}-\mathrm{XIII}{ }^{\mathrm{e}} \mathrm{s}$.) ou encore vers l'Italie avec la Maison angevine (XIv ${ }^{\mathrm{e}} \mathrm{s}$.)...

La quatrième section ( $«$ Religious Cults and Symbols of Power ») fait la part belle à la royauté hongroise et à la mise en scène du pouvoir à la fois par l'édification au sens littéral du terme (la construction hagiographique) mais également grâce aux images et aux ornements (G. Klaniczay, V. Lucherini et P. Lővei,). Ainsi, sont examinés le culte des saints rois et des saintes princesses, les sculptures et les effigies funéraires qui, se substituant aux corps disparus, affirment la pérennité du corps royal ou encore les objets-signes (couronnes, manteaux du couronnement). Au milieu du XIV ${ }^{e}$ s., la réécriture de l'histoire du royaume en images, sous les Angevins, fait l'objet d'un très stimulant développement à partir d'une analyse détaillée du Chronicon pictum.

Le cinquième chapitre ( $"$ Forms of Art Between Public and Private Use ») permet d'aborder la représentation élitaire (à travers les objets d'orfèvrerie, les textiles, la culture livresque) et la constitution des trésors ecclésiastiques (E. Wetter et A. Boreczky). Outre une réflexion sur les artisans, les ateliers, la circulation, les modes de production, les échanges et la commande artistique, les contributions soulignent aussi la manière dont ces objets, enjeux d'inventaires minutieux du Moyen Âge au XIX ${ }^{\mathrm{e}}$ s., glissent d'objets-signes - memorabilia ou substantialisant un pouvoir - à la définition d'un art national.

La dernière section de l'ouvrage, « The Middle Ages After the Middle Ages ", s'inscrit pleinement dans le projet scientifique détaillé dans l'introduction (I. Takàcs, A. Mikò et G. Gy Papp). Sans rien céder à la facilité téléologique, les développements sur la création artistique à la cour sous les règnes de Sigismond de Luxembourg et de Matthias Corvin soulignent les continuités entre l'art du Moyen Âge et celui du $\mathrm{XVI}^{\mathrm{e}} \mathrm{s}$. La troisième contribution évoque la réception du Moyen Âge au XIX ${ }^{\mathrm{e}} \mathrm{s}$. à travers le courant néo-gothique qui a traversé la création architecturale hongroise.

Deux annexes complètent cet ouvrage, la première, «Medieval Artworks and Monuments », et la seconde, "Museums and Collections Holding Medieval Art». Elles sont composées de notices sur des édifices et des œuvres visuelles majeurs ainsi que sur les musées et les collections d'art médiéval en Hongrie 
contemporaine. Ces notices sont conçues comme les articles qui forment le corps du livre : une approche historique, historiographique, une analyse et les références bibliographiques les plus significatives. Les présentations des musées et des collections font toujours l'objet d'une réflexion visant à articuler étroitement la question européenne et les fonctions patrimoniales de ces institutions.
À bien des égards, ce livre est un modèle, dépassant l'ouvrage de synthèse pour s'imposer comme une référence, désormais indispensable non seulement sur le sujet mais aussi pour tout médiéviste s'intéressant à la culture et à l'art du Moyen Âge occidental.

Cécile Voyer

UMR 7302 - CESCM, Université de Poitiers 